\title{
What is the origin of a diffuse IGM in compact groups?
}

\section{Verdes-Montenegro* ${ }^{\dagger}$}

Instituto de Astrofisica de Andalucia-CSIC

E-mail: lourdes@iaa.es

\section{S. Borthakur}

University of Massachussets

E-mail: sancheastro.umass.edu

\section{Yun}

University of Massachussets

E-mail: myuneastro.umass.edu

\section{A. del Olmo}

Instituto de Astrofisica de Andalucia-CSIC

E-mail: chony@iaa.es

\section{J. Perea}

Instituto de Astrofisica de Andalucia-CSIC

E-mail: jaime@iaa.es

Since the discovery of large HI deficiencies in Hickson Compact Groups (HCGs), the fate of atomic gas in these systems has been a mystery. We previously proposed an evolutionary scenario where the amount of $\mathrm{HI}$ decreases with the secular evolutionary state of a group. But what physical processes give rise to this decrease? Our X-ray study of 8 of the most HI deficient HCGs suggested that galaxy-IGM interaction is not the dominant mechanism driving cold gas out of group members (Rasmussen et al 2008). In order to explore this issue further, we have gathered VLA and GBT data for a complete distance-limited sample of 22 HCGs with normal, deficient, and highly deficient HI properties. The GBT observations (Borthakur et al 2010) revealed evidence for a significant $\mathrm{HI}$ excess compared with the interferometer measurements, and this excess appears to increase with the evolutionary phase of the group, possibly associated with the diffusing tidal debris in the IGM.

Here, we present the first detailed comparison of VLA \& GBT data in Stephan's Quintet (HCG 92) as a first step to examine the spatial and kinematic link between the high surface brightness to diffuse HI emission in these groups. Our preliminary results suggest that tidal tails could evolve to a diffuse neutral component, as suggested in Verdes-Montenegro et al (2001) and Borthakur et al (2010).

ISKAF2010 Science Meeting - ISKAF2010

June 10-14, 2010

Assen, the Netherlands

\footnotetext{
* Speaker.

${ }^{\dagger}$ LVM is partially supported by DGI Grant AYA 2005-07516-C02-01 and Junta de Andalucia (Spain).
} 


\section{HI deficiency in Hickson Compact Groups. Previous studies}

A combined analysis of single dish HI data for 72 HCGs and the VLA data for 16 HCGs has revealed that $60 \%$ of $\mathrm{HI}$ emission is missing in these groups, which is similar in magnitude to the HI deficiency found in Virgo and Coma clusters and Coma I group (Verdes-Montenegro et al 2001). Evidence for tidal interactions and tidal stripping is common, and the HI deficiency is larger in individual galaxies (76\%). So where is this missing gas? The fraction of HI deficient and nondeficient groups detected in the ROSAT survey is $45 \%$ and 18\%, respectively (Ponman et al 1996), and it was suggested that hot gas might have originated in cold gas stripped from the galaxies plus phase transition. However, ram pressure stripping seems insufficient to explain the observed HI deficiencies (Rasmussen et al 2008). Has past star formation exhausted the gas supply? Or could there exist an extended component stripped from the member galaxies which remains neutral in the intragroup medium?

In order to explore the latter possibility, we have performed a GBT study (Borthakur et al 2010) of a complete distance limited ( $<<100 \mathrm{Mpc}$ ) sample of 22 groups with at least 4 true members and at least one spiral. The upgraded GBT radiotelescope provides a high sensitivity ( $\mathrm{rms} /$ hour $=0.7 \mathrm{mJy}$; a 2-20 improvement with respect to previous observations) with an improved baseline stability and a large bandwidth $(\Delta \mathrm{v}=2500 \mathrm{~km} / \mathrm{s})$. The integrated HI spectra obtained from GBT are compared with the equivalent spectra synthesized by integrating the VLA data cubes in the area covered by GBT ( 8.5 arcminutes), and significant excess emission is found in the GBT spectra. Given the superior surface brightness sensitivity of the GBT, this excess is interpreted as originating from diffuse, low column density $\mathrm{HI}$ in these groups.

In Borthakur et al we proposed that the missing gas in the VLA data may exist as: (1) Low column density diffuse medium which can only be detected by GBT; (2) Spatially extended sheets, filaments, or extended disks that are missed by the VLA because of spatial filtering; or (3) A combination of both. The large inferred filling factor suggests that the excess gas is extended and occupy a significant fraction (median $=40 \%$ ) of the GBT beam. The broad spectral signatures specifically rule out cold discrete clumps or filaments. The excess gas mass fraction is the highest in the least HI-deficient groups. The excess gas content increases with the evolutionary phase of the group, and we proposed that faint diffuse neutral medium produced by the slow evolution of tidal debris accounts for this excess. According to the evolutionary scenario proposed in VerdesMontenegro et al (2001), the largest fraction of diffuse neutral IGM should be found in groups where the tidal debris has had enough time to evolve into an extended component. This is the scenario we want to test further here.

\section{GBT versus VLA integrated emission for Stephan's Quintet}

Stephan's Quintet is group 92 in Hickson Catalogue. A detailed description of the group members and notations, and the VLA HI observations are found in Sulentic et al (2001) and Williams et al (2002). The new GBT spectrum is included in Borthakur et al paper, and a detailed comparison between the VLA and single dish data presented here has not been performed previously. No HI emission is found at the position of the new intruder in the VLA data while emission features are found to the north (NW-LV) and south of the intruder position. Interestingly, the GBT spectrum 
shows emission in the velocity gap between those two components, suggesting that faint diffuse gas associated to NGC 7318b might still survive the harsh conditions. The NW-LV clump appears more extended in the VLA map than its more compact counterpart at the main group velocity, NW-HV. This difference is confirmed by the GBT spectrum. The majority of the HI missed by the VLA is associated to the velocity of the main system, which includes Arc-N and S and NW-HV. An inspection of the VLA data cube suggests that this missing gas results from the tidal features Arc-N and/or S being spread in a large area. Hints of faint but extended emission corresponding to the emission detected by the GBT might be present in the velocity range $6432-6518 \mathrm{~km} / \mathrm{s}$, where a higher level of "noise" is observed at a level of $2 \times 10^{18}$ atoms $\mathrm{cm}^{-2}$.

These results support the idea proposed in our previous works: the diffuse neutral component seems associated to the presence of evolved tidal tails. We plan to test this hypothesis further using the dataset we have built for our complete sample of compact groups.

\section{Perspectives for the golden age of radioastronomy}

Studies like the one proposed here will strongly benefit from the improvements provided by the most recent and forthcoming facilities, as, among others, large velocity coverage (e.g EVLA, WSRT), large field of view (e.g. Apertif, ASKAP), sensitivity to faint extended features (MeerKat) or large dynamic range (ASKAP).

\section{References}

[1] Borthakur, S., Yun, M. S., Verdes-Montenegro, L. 2010, ApJ 710385

[2] Ponman, T. J., Bourner, P. D. J., Ebeling, H., \& Bohringer, H. 1996, MNRAS 283, 690

[3] Rasmussen, J., Ponman, T. J., Verdes-Montenegro, L., Yun, M. S., \& Borthakur, S. 2008, MNRAS, 388,1245

[4] Sulentic, J. W., Rosado, M., Dultzin-Hacyan, D., Verdes-Montenegro, L., Trinchieri, G., Xu, C., \& Pietsch, W. 2001, AJ, 122, 2993

[5] Verdes-Montenegro, L., Yun, M. S., Williams, B. A., Huchtmeier, W. K., Del Olmo, A., \& Perea, J. 2001, A\&A 377, 812

[6] Williams, B. A., Yun, M. S., \& Verdes-Montenegro, L. 2002, AJ, 123, 2417 